\title{
SMR
}

\section{Genetic background of Escherichia coli isolates from peritoneal dialysis patients with peritonitis and uninfected control subjects}

\author{
Y.F. Li ${ }^{1,2 *}$, N. Su ${ }^{2 *}$, S.Y. Chen ${ }^{3}$, W.X. Hu' ${ }^{1}$, F.F. Li ${ }^{1}$, Z.P. Jiang ${ }^{1,2}$ and X.Q. Yu ${ }^{1}$ \\ ${ }^{1}$ Department of Nephrology, The First Affiliated Hospital, Sun Yat-Sen University, \\ Guangzhou, China \\ 2Department of Nephrology, The Sixth Affiliated Hospital, Sun Yat-Sen University, \\ Guangzhou, China \\ ${ }^{3}$ Department of Parasites, Guangzhou Disease Control and Prevention Center, \\ Guangzhou, China
}

*These authors contributed equally to this study.

Corresponding author: Z.P. Jiang

E-mail: zongpeijiang@yeah.net

Genet. Mol. Res. 15 (1): gmr.15017341

Received August 29, 2015

Accepted October 30, 2015

Published March 28, 2016

DOI http://dx.doi.org/10.4238/gmr.15017341

\begin{abstract}
Escherichia coli is the most common cause of Gram-negative peritonitis resulting in peritoneal function deterioration as well as poor clinical outcome in continuous ambulatory peritoneal dialysis (PD) patients. In this study, we analyzed the phylogenetic background and genetic profile of the $E$. coli isolates and sought to determine the characteristics of specific bacteria associated with peritonitis. E. coli isolates from 56 episodes of peritonitis in 46 PD patient cases and rectal isolates from 57 matched PD control patient cases were compared for both phylogenetic groups and the presence of virulence factors (VFs). There were no significant differences in terms of demographic data between the peritonitis and control groups. Peritonitis isolates exhibited a significantly greater prevalence of 8 VFs.
\end{abstract}


In multivariate logistic regression analysis, kpsMT II (group 2 capsule synthesis) was the strongest VF predictor of peritonitis (OR $=8.02 ; 95 \% \mathrm{Cl}$ = 3.18-20.25; $\mathrm{P}<0.001$ ), followed by traT (serum-resistance-associated outer membrane protein $)(\mathrm{OR}=3.83 ; 95 \% \mathrm{Cl}=1.33-11.03 ; \mathrm{P}=0.013)$. The pathogenic groups of $E$. coli contained a higher concentration of individual VFs compared to the commensal groups. The prevalence of pathogenic $E$. coli was much higher in peritoneal isolates than rectal isolates $(64.3$ vs $31.6 \%, \mathrm{P}=0.001)$. Our results indicate that the $E$. coli peritonitis and rectal isolates are different in PD patients. The specific VFs associated with peritonitis isolates may directly contribute to the pathogenesis of peritonitis.

Key words: Escherichia coli; Virulence factors; Peritonitis; Peritoneal dialysis

\section{INTRODUCTION}

Escherichia coli is the primary cause of Gram-negative bacteria peritonitis in patients with end-stage renal disease (ESRD) being treated by peritoneal dialysis (PD). It is the third to fourth most common cause of peritonitis, accounting for $3.83-10.5 \%$ of all peritonitis in PD patients (Krishnan et al., 2002; Chow et al., 2005; Mujais, 2006; Kotsanas et al., 2007; Ghali et al., 2011). PD-related peritonitis is considered a common and severe complication that results in prolonged hospitalization, requiring catheter removal, discontinuing hemodialysis, or even mortality (Ghali et al., 2011). Some studies (Choi et al., 2004) have found that E. coli as an agent of refractory peritonitis can cause poor clinical outcomes in a high proportion of cases.

E. coli can be classified into 3 broad clinical categories: commensal strains, intestinal pathogenic strains, and extra-intestinal pathogenic strains (Russo and Johnson, 2000). They can also be categorized by multilocus enzyme electrophoresis results into four main phylogenetic groups, denominated as A, B1, B2, and D (Herzer et al., 1990). Extra-intestinal pathogenic strains belong mostly to groups $\mathrm{B} 2$ and $\mathrm{D}$, while commensal $E$. coli strains mainly belong to groups $A$ and B1 (Picard et al., 1999). Strains causing extra-intestinal infections contain various virulence factors (VFs) including adhesions, toxins, siderophores, polysaccharide coatings, and some other invasions and protections (Picard et al., 1999; Duriez et al., 2001; Johnson and Russo, 2002). In previous studies (Sannes et al., 2004; Johnson et al., 2005; Sánchez et al., 2008; Mabbett et al., 2009; Bert et al., 2008, 2010), the phylogenetic background and virulence profiles of pathogenic isolates have been investigated from patients with urinary tract infections, bacteremia, spontaneous bacterial peritonitis (SBP) or coeliac disease. However, the specific characteristics of E. coli in peritonitis isolates infecting PD-treated patients and their potential in the pathogenesis and prognosis of peritonitis remain undefined. Increasing evidence suggests that PD-related peritonitis caused by Enterobacteriaceae resulted from bacterial translocation from the intestinal lumen to the peritoneal cavity (Szeto et al., 2005, 2006; Chuang et al., 2009). Other than host factors from patients themselves, bacterial factors may also play important roles in the development of PDrelated peritonitis. Therefore, the identification of $E$. coli characteristics from isolates would be helpful to understand the pathogenesis of PD-related E. coli peritonitis.

Fifty-six ascites isolates from continuous ambulatory peritoneal dialysis (CAPD) patients with peritonitis and 57 rectal isolates from matched patients without peritonitis were examined in this study. We analyzed and compared the phylogenetic background and virulence profiles of $E$. 
coli between these two groups. Additionally, we sought to examine the relationships between the characteristics of these bacteria in peritonitis.

\section{MATERIAL AND METHODS}

\section{Isolates from patients}

The database files of the Clinical Microbiology Laboratory and the medical records of patients in the Department of Nephrology were reviewed in order to identify episodes of $E$. coli peritonitis that occurred in CAPD patients at the First Affiliated Hospital of Sun-Yat San University (SYSU) between December 1, 2008 and November 30, 2011. Peritonitis was defined as having the clinical features of peritonitis (cloudy dialysate or abdominal pain) and dialysate leukocytosis (white blood cell count $>100 / \mu \mathrm{L}$ with $>50 \%$ neutrophils). Relapsing peritonitis was defined as an episode that occurs within 4 weeks of completion of therapy of a prior episode with the same individual. Repeat-peritonitis was defined as an episode that occurs more than 4 weeks after the completion of therapy of a prior episode with the same individual (Piraino et al., 2005; Li et al., 2010). Relapsing and polymicrobial peritonitis were excluded from the present study. Finally, a collection of 56 ascites isolates of $E$. coli were obtained from 56 episodes of peritonitis that occurred in 46 patients, 4 of whom had 1 repeated episode and 3 had 2 repeated episodes.

During the study period, $50-\mathrm{mL}$ effluent samples from patients with PD-related peritonitis were centrifuged at $3000 \mathrm{~g}$ for $15 \mathrm{~min}$ at room temperature. The pellet was resuspended in 10 $\mathrm{mL}$ supernatant and a set of aerobic and anaerobic blood culture bottles was inoculated with a $5-\mathrm{mL}$ sample. The whole process was aseptically performed. The blood culture system used was BactAlert (bioMérieux, Marcy-l'étoile, France). Bottles were incubated at $37^{\circ} \mathrm{C}$ for $3-5$ days. $E$. coli was identified by the VITEK 2 system (bioMérieux). For the control group, 57 rectal isolates were obtained from matched CAPD patients without peritonitis in our PD Center before sample collection and lacked evidence of acute infection. Rectal swabs were collected and processed to isolate E. coli as described previously (Johnson et al., 1998), and one arbitrarily selected E. coli colony per sample was analyzed (Sannes et al., 2004).

Peritonitis and rectal isolates were stored at $-80^{\circ} \mathrm{C}$ in $15 \%$ glycerol until subsequent analysis. Informed consent forms were obtained from patients who participated in the present study, which was approved by the Ethics Committee of the First Affiliated Hospital of SYSU. The clinical data of the patients and control subjects were retrieved from the medical files in the PD center, including age, gender, PD duration, and primary etiology of renal failure.

\section{DNA isolation, phylogenetic group analysis and virulence gene detection}

E. coli isolates were incubated on Luria-Bertani (LB) agar plates overnight at $37^{\circ} \mathrm{C}$. Single colonies were picked and further incubated in $7 \mathrm{~mL}$ LB broth at $37^{\circ} \mathrm{C}$ with shaking at $120 \mathrm{rpm}$ for $12 \mathrm{~h}$. Subsequently, DNA was extracted using the QIAGEN DNeasy tissue kit (QIAGEN, USA) according to the manufacturer protocol. The phylogenetic groups (A, B1, B2, or D) of the isolates was determined by triplex polymerase chain reaction (PCR) as previously described (Clermont et al., 2000). Virulence genes encoding 31 extra-intestinal VFs of E. coli were tested using multiplex PCR assays as described elsewhere (Johnson and Stell, 2000; Chapman et al., 2006). All PCRs were carried out in duplicate. The VFs included 13 adhesins (papAH, papC, papEF, papG, sfa/foc, sfaS, focG, afa/draBC, iha, gafD, nfaE, bmaE, fimH), 3 toxins (hlyA, cnf1, cdtB), 4 siderophores 
(iutA, fyuA, iroN, ireA), 5 capsule synthesis genes (kpsMT II, kpsMT K1, kpsMT K5, kpsMT III, rfc), and 6 miscellaneous traits (cvaC, ibeA, traT, ompT, iss, $P A I)$. PapG was also detected with alleles I, II and III. The total number of VF genes (including papG alleles) for which the isolate was positive was determined as the VF score (Johnson et al., 2002).

\section{Statistical analysis}

Statistical analyses were carried out with the SPSS software 13.0 (Chicago, IL, USA). Comparisons of categorical variables were made by the use of either a Pearson chi-square test or a Fisher exact test (2-tailed). Comparisons of categorical variables and VF scores (the sum of all VFs from an individual isolate) were done by the use of the Wilcoxon rank sum test. Virulence factors independently related to peritonitis were analyzed by the use of stepwise multivariate logistic regression. The threshold for statistical significance was established at $\mathrm{P} \leq 0.05$.

\section{RESULTS}

\section{Host characteristics}

Patients with peritonitis and control subjects were similar in terms of their age, gender, PD duration and primary kidney disease (Table 1).

\section{Table 1. Host characteristics of PD patients with or without peritonitis.}

\begin{tabular}{l|c|c|c}
\hline Host characteristics & Patients with peritonitis $(\mathrm{N}=46)$ & Patients without peritonitis (N = 57) & P value \\
\hline Age (years) & $49.5 \pm 16.2$ & $50.5 \pm 14.7$ & 0.75 \\
\hline Male gender [No. (\%)] & $31(67.4)$ & $40(70.2)$ & 0.62 \\
\hline PD duration (month) & $16.9 \pm 17.1$ & $17.4 \pm 13.6$ & 0.88 \\
\hline Primary kidney disease & $30(65.2)$ & $31(54.4)$ & \\
\hline CGN [No. (\%)] & $6(13.1)$ & $9(15.8)$ & 0.27 \\
\hline Hypotension [No. (\%)] & $3(6.5)$ & $7(12.3)$ & 0.69 \\
\hline Diabetes [No. (\%)] & $3(6.5)$ & $3(5.2)$ & 0.33 \\
\hline PKD [No. (\%)] & $4(8.7)$ & $7(12.3)$ & 0.79 \\
\hline Other & & & 0.56 \\
\hline
\end{tabular}

$\mathrm{PD}=$ peritoneal dialysis; $\mathrm{CGN}=$ chronic glomerulonephritis; $\mathrm{PKD}=$ polycystic kidney disease .

\section{Phylogenetic group}

The two groups (peritonitis and control) differed significantly in the prevalence of phylogenetic groups B1 and B2 but not group A. Group D was more prevalent in peritonitis isolates compared to control although this was not statistically significant $(P=0.084$; Table 2$)$. Group $D$ was the most prevalent in the peritonitis isolates, while group $A$ was most prevalent among the rectal (control) isolates (Table 2). When combining the phylogenetic virulent groups B2 and D and the commensal groups A and B1, the virulent groups (B2 plus D) were observed to be significantly associated with peritonitis (Table 2).

\section{Virulence characteristics}

Of the $31 \mathrm{VFs}$ examined, all but gafD (G fimbriae), cdtB (cytolethal distending toxin) and $k p s M T$ III (group 3 capsule synthesis) were detected in at least 1 isolate from each group 
(Table 2). Expect for 2 rectal isolates, the remaining 111 strains contained $\geq 1$ VF. Compared with the 57 rectal isolates from the control subjects, the 56 peritonitis isolates from the case patients exhibited a statistically higher prevalence of 8 VFs, including 1 adhesin [afa/draBC (Dr-binding adhesins)], 2 capsule markers [kpsM II, kpsM K5 (kpsM II variant)], 2 iron-acquisition factors [fyuA (yersiniabactin receptor) and iutA (aerobactin system)], and 3 miscellaneous VFs [traT (serumresistance-associated outer membrane protein), ibeA (invasion of brain endothelium A) and PAI (pathogenicity-associated island)] (Table 3). On the contrary, not one VF was more prevalent among the rectal isolates than the peritonitis isolates (Table 3). Consequently, aggregate VF scores were statistically higher among the peritonitis isolates (mean $=8.7$; range $=1-20$ ) than among the rectal isolates $($ mean $=5.4$; range $=0-17)($ Table 4$)$.

Table 2. Phylogenetic distribution of peritonitis and rectal Escherichia coli isolates.

\begin{tabular}{l|c|c|c}
\hline \multirow{2}{*}{ Phylogenetic group (No. of isolates) } & \multicolumn{2}{|c|}{ Prevalence of phylogenetic group, [No. (\%) of isolates] } & \multirow{2}{*}{$P$ value } \\
\cline { 2 - 3 } & Peritonitis isolates $(\mathrm{N}=56)$ & Rectal isolates (N = 57) & 0.194 \\
\hline A (41) & $17(30.4)$ & $24(42.1)$ & 0.002 \\
\hline B1 (18) & $3(5.3)$ & $15(26.3)$ & 0.015 \\
\hline B2 (22) & $16(28.6)$ & $6(10.5)$ & 0.084 \\
\hline B2 or D (54) & $20(35.7)$ & $12(21.1)$ & 0.001 \\
\hline
\end{tabular}

No isolates exhibited gafD (G fimbriae), cdtB (cytolethal distending toxin) or kpsMT III (group 3 capsule synthesis). Afa/draBC, Dr-binding adhesins; bmaE, M fimbriae; cnf1, cytotoxic necrotizing factor 1; cvaC, colicin (microcin) $\mathrm{V}$; fimH, type I fimbriae; focG, F1C fimbriae; fyuA, yersiniabactin receptors; hlyA, a-Hemolysin; ibeA, invasion of brain endothelium $\mathrm{A}$; iha, putative adhesin-siderophore; ire $A$ and iron, novel siderophore receptors; iss, increased serum survival; iutA, aerobactin system; K1, K1 kpsMT II variant; K5, K5 kpsMT II variant; kpsMT II, group 2 capsule synthesis; $n f a E$, non-fimbrial adhesin I assembly and transport; ompT, outer membrane protein T; $P A I$, pathogenicity-associated island; $p a p A H, \mathrm{P}$ fimbriae structural subunit; $p a p C, \mathrm{P}$ fimbriae chaperone; $p a p E F, P$ fimbriae tip pilins; $p a p G, P$ fimbrial adhesion molecule, with variants I, II and III; rfc, O4 lipopolysaccharide synthesis; sfa/foc, S and F1C fimbriae; sfaS, S fimbrial adhesin; traT, serum-resistance-associated outer membrane protein.

\section{Phylogenetic distribution of VFs}

In the present study, peritonitis isolates contained a higher prevalence of VFs than the rectal isolates. In addition, phylogenetic virulent groups (B2 plus D) had a higher prevalence among peritonitis isolates. Therefore, we analyzed the difference in prevalence of VFs between virulent groups (B2 plus D) and commensal groups (A plus B1). Significant differences in prevalence favoring virulent groups (B2 plus D) over commensal groups (A plus B1) were noted for 6 adhesins (papAH, papC, papG, papG with allele I or II, iha, afa/draBC, fimH), all of the iron-acquisition factors (iroN, fyuA, ire $A$, and iutA), one toxin ( $h l y D), 3$ capsule markers (kpsM II, kpsM K1 and kpsM K5), and 5 miscellaneous VFs [CvaC, ibeA (invasion of brain endothelium A), traT, ompT, $P A$ ]. As a result, the mean VF scores among phylogenetic groups differed considerably. As shown in Table 4, the mean VF scores were the highest in group B2, followed by group D, and groups $A$ and B1 displayed the lowest mean VF scores (Table 5). 
Table 3. Virulence factors (VFs) of peritonitis and rectal Escherichia coli isolates.

\begin{tabular}{|c|c|c|c|}
\hline \multirow{2}{*}{ Virulence factor genes } & \multicolumn{2}{|c|}{ Prevalence of VFs [No. (\%)] } & \multirow{2}{*}{$P$ value } \\
\hline & Peritonitis isolates $(N=56)$ & Rectal isolates $(\mathrm{N}=57)$ & \\
\hline \multicolumn{4}{|l|}{ Adhesins } \\
\hline рарAH & $11(20)$ & $6(11)$ & 0.175 \\
\hline papC & $11(20)$ & $9(16)$ & 0.592 \\
\hline papEF & $13(23)$ & $8(14)$ & 0.21 \\
\hline papG & $11(20)$ & $7(12)$ & 0.285 \\
\hline papG allele I & $5(9)$ & $2(4)$ & 0.271 \\
\hline papG allele II & $8(14)$ & $5(9)$ & 0.358 \\
\hline papG allele III & $2(4)$ & $2(4)$ & 1 \\
\hline Sfa/focDE & $3(5)$ & $8(14)$ & 0.12 \\
\hline sfas & $2(4)$ & $1(2)$ & 0.618 \\
\hline foc $G$ & 0 & $3(5)$ & 0.243 \\
\hline Iha & $22(39)$ & $15(26)$ & 0.142 \\
\hline$a f a / d r a B C$ & $12(21)$ & $4(7)$ & 0.028 \\
\hline bmaE & 0 & $2(4)$ & 0.496 \\
\hline$n f a E$ & $1(2)$ & 0 & 0.496 \\
\hline $\mathrm{fimH}$ & $47(84)$ & $52(91)$ & 0.239 \\
\hline \multicolumn{4}{|l|}{ Toxins } \\
\hline hlyA & $1(2)$ & $4(7)$ & 0.364 \\
\hline cnf1 & $1(2)$ & $1(2)$ & 1 \\
\hline \multicolumn{4}{|l|}{ Capsule synthesis } \\
\hline kpsMT II & $45(80)$ & $15(26)$ & $<0.001$ \\
\hline kpsMT K1 & $19(34)$ & $13(23)$ & 0.190 \\
\hline kpsMT K5 & $26(46)$ & $6(11)$ & $<0.001$ \\
\hline$r f c$ & $2(4)$ & $1(2)$ & 0.618 \\
\hline \multicolumn{4}{|l|}{ Siderophores } \\
\hline iutA & $45(80)$ & $24(42)$ & $<0.001$ \\
\hline fyuA & $47(84)$ & $29(51)$ & $<0.001$ \\
\hline iron & $15(27)$ & $12(21)$ & 0.475 \\
\hline ireA & $8(14)$ & $5(9)$ & 0.358 \\
\hline \multicolumn{4}{|l|}{ Miscellaneous } \\
\hline$c v a C$ & $13(23)$ & $7(12.3)$ & 0.128 \\
\hline$i b e A$ & $6(11)$ & 0 & 0.013 \\
\hline $\operatorname{traT}$ & $49(88)$ & $28(49)$ & $<0.001$ \\
\hline ompT & $29(52)$ & $20(35)$ & 0.073 \\
\hline iss & $10(18)$ & $9(16)$ & 0.769 \\
\hline$P A I$ & $25(45)$ & $8(14)$ & $<0.001$ \\
\hline
\end{tabular}

Only those VFs yielding a significant phylogenetic association $(P \leq 0.05)$ are shown. No isolate exhibited gafD (G fimbriae), cdtB (cytolethal distending toxin), or kpsMT III (group 3 capsule synthesis). No significant association with phylogenetic group was seen for bmaE (M fimbriae), cnf1 (cytotoxic necrotizing factor 1), focG (F1C fimbriae), iss (increased serum survival), nfaE (nonfimbrial adhesin I assembly and transport), papEF (P fimbriae tip pilins), variants III of papG (P fimbrial adhesion molecule), rfc (O4 lipopolysaccharide synthesis), sfa/foc (S and F1C fimbriae), 
sfaS (S fimbrial adhesin). afa/draBC, Dr-binding adhesins; cvaC, colicin (microcin) V; fimH, type I fimbriae; fyuA, yersiniabactin receptor; hlyA, a-Hemolysin; $i b e A$, invasion of brain endothelium $\mathrm{A}$; iha, putative adhesin-siderophore; ire $A$ and iron, novel siderophore receptors; iut $A$, aerobactin system; K1, K1 kpsMT II variant; K5, K5 kpsMT II variant; kpsMT II, group 2 capsule synthesis; ompT, outer membrane protein $\mathrm{T}$; $P A I$, pathogenicity-associated island; $p a p A H, \mathrm{P}$ fimbriae structural subunit; $p a p C, \mathrm{P}$ fimbriae chaperone; $p a p G, \mathrm{P}$ fimbrial adhesion molecule, with variants I and II; traT, serum-resistance-associated outer membrane protein.

Table 4. Virulence factor (VF) scores for peritonitis and rectal Escherichia coli isolates, by phylogenetic group

\begin{tabular}{l|c|c|c|c}
\hline \multirow{2}{*}{ Phylogenetic group } & \multicolumn{3}{|c|}{ Mean VF score (No.) } & \multirow{2}{*}{ P value (peritonitis vs rectal isolates) } \\
\cline { 2 - 4 } & $\begin{array}{c}\text { All isolates } \\
(\mathrm{N}=113)\end{array}$ & $\begin{array}{c}\text { Peritonitis isolates } \\
(\mathrm{N}=56)\end{array}$ & $\begin{array}{c}\text { Rectal isolates } \\
(\mathrm{N}=57)\end{array}$ & $<.4$ \\
\hline Total & 7 & 8.7 & $3.3(24)^{\mathrm{a}, \mathrm{b}}$ & 0.001 \\
\hline Group A & $4.3(41)^{\mathrm{a}, \mathrm{b}}$ & $5.7(17)^{\mathrm{a}, \mathrm{c}}$ & $3.4(15)^{\mathrm{a}, \mathrm{b}}$ & 0.005 \\
\hline Group B1 & $3.9(18)^{\mathrm{a}, \mathrm{b}}$ & $6.7(3)$ & $11.7(6)$ & 0.529 \\
\hline Group B2 & $12.6(22)$ & $13(16)$ & $8.9(12)$ & 0.610 \\
\hline Group D & $8.5(32)^{\mathrm{a}}$ & $8.2(20)^{\mathrm{a}}$ & \\
\hline
\end{tabular}

aP $\leq 0.001$ for indicated group $v s$ group B2 within column. ${ }^{\mathrm{b}} \mathrm{P} \leq 0.001$ for indicated group $v s$ group $\mathrm{D}$ within column. ${ }^{\mathrm{c} P}$ $<0.01$ for indicated group vs group D within column.

Table 5. Phylogenetic distribution of virulent factors (VFs) among peritonitis and rectal Escherichia coli isolates.

\begin{tabular}{|c|c|c|c|}
\hline \multirow{2}{*}{ Virulence factor genes } & \multicolumn{2}{|c|}{ Prevalence of VFs [No. (\%)] } & \multirow{2}{*}{$P$ value } \\
\hline & $A+B 1(N=59)$ & $B 2+D(N=54)$ & \\
\hline \multicolumn{4}{|l|}{ Adhesins } \\
\hline рарАH & $2(3)$ & $15(28)$ & $<0.001$ \\
\hline papC & $2(3)$ & $18(33)$ & $<0.001$ \\
\hline papG & $1(2)$ & $17(32)$ & $<0.001$ \\
\hline papG allele I & 0 & $7(13)$ & 0.005 \\
\hline papG allele II & 0 & $13(24)$ & $<0.001$ \\
\hline iha & $12(20)$ & $25(46.3)$ & 0.003 \\
\hline$a f a / d r a B C$ & $3(5)$ & $13(24)$ & 0.004 \\
\hline $\mathrm{fimH}$ & $48(81)$ & $51(94)$ & 0.035 \\
\hline \multicolumn{4}{|l|}{ Toxins } \\
\hline hlyA & 0 & $5(9)$ & 0.023 \\
\hline \multicolumn{4}{|l|}{ Capsule synthesis } \\
\hline kpsMT II & $17(29)$ & $43(80)$ & $<0.001$ \\
\hline kpsMT K1 & $10(17)$ & $22(41)$ & 0.005 \\
\hline kpsMT K5 & $9(15)$ & $23(43)$ & 0.001 \\
\hline \multicolumn{4}{|l|}{ Siderophores } \\
\hline iutA & $25(42)$ & $44(82)$ & $<0.001$ \\
\hline fyuA & $28(48)$ & $48(90)$ & $<0.001$ \\
\hline iroN & $9(15)$ & $18(33)$ & 0.024 \\
\hline ireA & $1(2)$ & $12(22)$ & 0.001 \\
\hline \multicolumn{4}{|l|}{ Miscellaneous } \\
\hline cvaC & $6(10)$ & $14(26)$ & 0.028 \\
\hline$i b e A$ & 0 & $6(11)$ & 0.01 \\
\hline $\operatorname{traT}$ & $32(54)$ & $45(83)$ & 0.001 \\
\hline ompT & $15(25)$ & $34(63)$ & $<0.001$ \\
\hline$P A I$ & $1(2)$ & $32(59)$ & $<0.001$ \\
\hline Overall VF score & 4.2 & 10.2 & $<0.001$ \\
\hline
\end{tabular}




\section{Multivariate logistic regression analysis}

Considering the relationships between the VFs and phylogenetic groups described above, multivariate logistic regression analysis was used to differentiate peritonitis isolates from rectal isolates. Two different models were constructed: the candidate predictor variables in the first model included only the 8 VFs, which were significantly higher in prevalence in the peritonitis isolates, while the second model also included phylogenetic groups. Whichever model was used, there were only 2 VFs significantly predicted for peritonitis, kpsMT II (group 2 capsule synthesis) and traT (serum-resistance-associated outer membrane protein). Of the two, kpsMT II was a stronger predictor than traT (Table 6).

Table 6. Stepwise multivariate logistic regression analysis for predictors of peritonitis.

\begin{tabular}{l|c|c}
\hline Candidate predictor variables, asignificant predictor variable (s) & OR $(95 \% \mathrm{Cl})$ & $\mathrm{P}$ value \\
\hline$k p s M T$ II & $8.02(3.18-20.25)$ & $<0.001$ \\
\hline $\operatorname{traT}$ & $3.83(1.33-11.03)$ & 0.013 \\
\hline
\end{tabular}

$\mathrm{Cl}=$ confidence interval; $\mathrm{OR}=$ odds ratio. ${ }^{a}$ Predictor variables in the regression model included phylogenetic groups [(B2 + D) compared with $(A+B 1)]$ and the virulence factors (VFs) below: afa/draBC, Dr-binding adhesins; fyuA, yersiniabactin receptor; iut $A$, aerobactin system; ibeA, invasion of brain endothelium A; K5, K5 kpsMT II variant; kpsMTII, group 2 capsule synthesis; $P A I$, pathogenicity-associated island; traT, serum-resistance-associated outer membrane protein.

\section{DISCUSSION}

To the best of our knowledge, this is the first study to compare the genetic structure and virulence-associated genes of $E$. coli from peritonitis isolates with rectal isolates from patients with ESRD treated with CAPD. In the present study, the case patients and uninfected control subjects from whom the studied isolates were derived were geographically, demographically, and temporally matched. We found that peritonitis isolates exhibited higher prevalence of phylogenetic group (B2 plus D) and some VFs compared to the rectal isolates. These findings demonstrated that the peritonitis isolates exhibit a superior virulence capability and the virulence properties may relate to the bacterial translocation that result in peritonitis.

The phylogenetic groups A, B1, B2, and D were found in different proportions in peritonitis isolates from case patients and rectal isolates from uninfected control subjects. Although none of the 4 groups were overwhelmingly dominant both in peritonitis and rectal isolates, the virulent groups (B2 and D) were more common in peritonitis isolates, while commensal groups ( $A$ and $B 1$ ) were more common in rectal isolates. The prevalence of virulent groups of $E$. coli among peritonitis isolates $(64 \%)$ is similar to what was previously reported for SBP or PD-related peritonitis isolates in Taiwan (54\%) (Wang et al., 2009) and SBP isolates in France (72\%) (Bert et al., 2010). However, the prevalence of virulent groups among pathogenic isolates in this study was lower than what was identified for urinary tract infection isolates and bacteremia isolates (Sannes et al., 2004; Johnson et al., 2005; Mabbett et al., 2009). As for the rectal isolates from PD patients without peritonitis in the present study, the distribution of phylogenetic groups was similar to that reported for isolates from healthy human populations in Europe (40\% A, 34\% B1, 11\% B2, and 15\% D). 
For each of the capsule synthesis, siderophores or miscellaneous, 2 or more VF factors were more frequent in peritonitis isolates than in rectal isolates but only one of the 12 adhesins investigated were more frequent in peritonitis isolates. There was no difference in prevalence of any toxin between the peritonitis and rectal isolates. In fact, most of the adhesins and all the toxins detected were uncommon in both the peritonitis and rectal isolates from the PD patients in this study. Finally, by multivariate logistic regression analysis, kpsMT II was found to be the VF most strongly associated with peritonitis, regardless as to whether the phylogenetic background was included or excluded in the model. This gene had been previously identified as an independent predictor for E. coli urinary-source bacteremia in American hospitalized patients (Marschall et al., 2012). Our findings indicate that further investigation of $k p s M T$ II is needed. In addition to the occurrence of the infection, virulence factors specific to the implicated strain may also be associated with the outcome. Thus, realizing the role of bacterial characteristics in the prognosis of PD-related peritonitis will be the next step of our study.

E. coli strains vary in the presence of VF genes with VF scores ranging from 0 to 20 , which is demonstrated by our results from both isolates. The VF scores gradually increased from group A plus B1 to group D to group B2. This finding was consistent with the result of $E$. coli isolates causing bacteremia or SBP reported previously (Sannes et al., 2004; Bert et al., 2008). The strength of the present study is that the cases and control patients were demographically, geographically and temporally similar. The limitations included a small sample size and all subjects were from a confined location, leading to lack of diversity in E. coli strains.

In conclusion, we found that $E$. coli characteristics varied considerably between ESRD patients treated with CAPD with peritonitis compared to without peritonitis. The genetic characteristics of certain E. coli strains seemed to be important in predicting pathogenic potential. The roles of some specific VFs associated with peritonitis isolates appear to play a role in the pathogenesis of peritonitis and warrant further investigation.

\section{Conflicts of interest}

The authors declare no conflict of interest.

\section{ACKNOWLEDGMENTS}

We wish to thank all doctors and nurses of the PD center from the First Affiliated Hospital of SYSU for collecting and providing patient clinical data, and all members of the microbiology laboratory of the First Affiliated Hospital of SYSU for providing the peritonitis isolates. Research supported by the Baxter Extramural Grant Program (\#2011CB504000).

\section{REFERENCES}

Bert F, Panhard X, Johnson J, Lecuyer H, et al. (2008). Genetic background of Escherichia coli isolates from patients with spontaneous bacterial peritonitis: relationship with host factors and prognosis. Clin. Microbiol. Infect. 14: 1034-1040.http:// dx.doi.org/10.1111/j.1469-0691.2008.02088.x

Bert F, Johnson JR, Ouattara B, Leflon-Guibout V, et al. (2010). Genetic diversity and virulence profiles of Escherichia coli isolates causing spontaneous bacterial peritonitis and bacteremia in patients with cirrhosis. J. Clin. Microbiol. 48: 27092714.http://dx.doi.org/10.1128/JCM.00516-10

Chapman TA, Wu XY, Barchia I, Bettelheim KA, et al. (2006). Comparison of virulence gene profiles of Escherichia coli strains isolated from healthy and diarrheic swine. Appl. Environ. Microbiol. 72: 4782-4795.http://dx.doi.org/10.1128/AEM.02885-05 
Choi P, Nemati E, Banerjee A, Preston E, et al. (2004). Peritoneal dialysis catheter removal for acute peritonitis: a retrospective analysis of factors associated with catheter removal and prolonged postoperative hospitalization. Am. J. Kidney Dis. 43: 103-111.http://dx.doi.org/10.1053/j.ajkd.2003.08.046

Chow KM, Szeto CC, Leung CB, Kwan BC, et al. (2005). A risk analysis of continuous ambulatory peritoneal dialysis-related peritonitis. Perit. Dial. Int. 25: 374-379.

Chuang YW, Shu KH, Yu TM, Cheng CH, et al. (2009). Hypokalaemia: an independent risk factor of Enterobacteriaceae peritonitis in CAPD patients. Nephrol. Dial. Transplant. 24: 1603-1608.http://dx.doi.org/10.1093/ndt/gfn709

Clermont O, Bonacorsi S and Bingen E (2000). Rapid and simple determination of the Escherichia coli phylogenetic group. Appl. Environ. Microbiol. 66: 4555-4558.http://dx.doi.org/10.1128/AEM.66.10.4555-4558.2000

Duriez P, Clermont O, Bonacorsi S, Bingen E, et al. (2001). Commensal Escherichia coli isolates are phylogenetically distributed among geographically distinct human populations. Microbiology 147: 1671-1676.http://dx.doi.org/10.1099/00221287147-6-1671

Ghali JR, Bannister KM, Brown FG, Rosman JB, et al. (2011). Microbiology and outcomes of peritonitis in Australian peritoneal dialysis patients. Perit. Dial. Int. 31: 651-662.http://dx.doi.org/10.3747/pdi.2010.00131

Herzer PJ, Inouye S, Inouye M and Whittam TS (1990). Phylogenetic distribution of branched RNA-linked multicopy singlestranded DNA among natural isolates of Escherichia coli. J. Bacteriol. 172: 6175-6181.

Johnson JR and Stell AL (2000). Extended virulence genotypes of Escherichia coli strains from patients with urosepsis in relation to phylogeny and host compromise. J. Infect. Dis. 181: 261-272.http://dx.doi.org/10.1086/315217

Johnson JR and Russo TA (2002). Extraintestinal pathogenic Escherichia coli: "the other bad E. coli". J. Lab. Clin. Med. 139: 155-162.http://dx.doi.org/10.1067/mlc.2002.121550 PubMed

Johnson JR, Brown JJ, Carlino UB and Russo TA (1998). Colonization with and acquisition of uropathogenic Escherichia coli as revealed by polymerase chain reaction-based detection. J. Infect. Dis. 177: 1120-1124.http://dx.doi.org/10.1086/517409

Johnson JR, Kuskowski MA, O'Bryan TT and Maslow JN (2002). Epidemiological correlates of virulence genotype and phylogenetic background among Escherichia coli blood isolates from adults with diverse-source bacteremia. J. Infect. Dis. 185: 1439-1447.http://dx.doi.org/10.1086/340506

Johnson JR, Owens K, Gajewski A and Kuskowski MA (2005). Bacterial characteristics in relation to clinical source of Escherichia coli isolates from women with acute cystitis or pyelonephritis and uninfected women. J. Clin. Microbiol. 43: 6064-6072.http://dx.doi.org/10.1128/JCM.43.12.6064-6072.2005

Kotsanas D, Polkinghorne KR, Korman TM, Atkins RC, et al. (2007). Risk factors for peritoneal dialysis-related peritonitis: can we reduce the incidence and improve patient selection? Nephrology (Carlton) 12: 239-245.http://dx.doi.org/10.1111/ j.1440-1797.2006.00756.x

Krishnan M, Thodis E, Ikonomopoulos D, Vidgen E, et al. (2002). Predictors of outcome following bacterial peritonitis in peritoneal dialysis. Perit. Dial. Int. 22: 573-581.

Li PK, Szeto CC, Piraino B, Bernardini J, et al.; International Society for Peritoneal Dialysis (2010). Peritoneal dialysis-related infections recommendations: 2010 update. Perit. Dial. Int. 30: 393-423.http://dx.doi.org/10.3747/pdi.2010.00049

Mabbett AN, Ulett GC, Watts RE, Tree JJ, et al. (2009). Virulence properties of asymptomatic bacteriuria Escherichia coli. Int. J. Med. Microbiol. 299: 53-63.http://dx.doi.org/10.1016/j.ijmm.2008.06.003

Marschall J, Zhang L, Foxman B, Warren DK, et al.; CDC Prevention Epicenters Program (2012). Both host and pathogen factors predispose to Escherichia coli urinary-source bacteremia in hospitalized patients. Clin. Infect. Dis. 54: 1692-1698. http://dx.doi.org/10.1093/cid/cis252

Mujais S (2006). Microbiology and outcomes of peritonitis in North America. Kidney Int. Suppl. 70: S55-S62.http://dx.doi. org/10.1038/sj.ki.5001916 PubMed

Picard B, Garcia JS, Gouriou S, Duriez P, et al. (1999). The link between phylogeny and virulence in Escherichia coli extraintestinal infection. Infect. Immun. 67: 546-553.

Piraino B, Bailie GR, Bernardini J, Boeschoten E, et al.; ISPD Ad Hoc Advisory Committee (2005). Peritoneal dialysis-related infections recommendations: 2005 update. Perit. Dial. Int. 25: 107-131. PubMed

Russo TA and Johnson JR (2000). Proposal for a new inclusive designation for extraintestinal pathogenic isolates of Escherichia coli: ExPEC. J. Infect. Dis. 181: 1753-1754.http://dx.doi.org/10.1086/315418

Sánchez E, Nadal I, Donat E, Ribes-Koninckx C, et al. (2008). Reduced diversity and increased virulence-gene carriage in intestinal enterobacteria of coeliac children. BMC Gastroenterol. 8: 50.http://dx.doi.org/10.1186/1471-230X-8-50

Sannes MR, Kuskowski MA, Owens K, Gajewski A, et al. (2004). Virulence factor profiles and phylogenetic background of Escherichia coli isolates from veterans with bacteremia and uninfected control subjects. J. Infect. Dis. 190: 2121-2128. http://dx.doi.org/10.1086/425984

Szeto CC, Chow KM, Kwan BC, Leung CB, et al. (2005). Hypokalemia in Chinese peritoneal dialysis patients: prevalence and prognostic implication. Am. J. Kidney Dis. 46: 128-135.http://dx.doi.org/10.1053/j.ajkd.2005.03.015

Szeto CC, Chow VC, Chow KM, Lai RW, et al. (2006). Enterobacteriaceae peritonitis complicating peritoneal dialysis: a review of 210 consecutive cases. Kidney Int. 69: 1245-1252.http://dx.doi.org/10.1038/sj.ki.5000037

Wang MC, Tseng CC, Wu AB, Huang JJ, et al. (2009). Different roles of host and bacterial factors in Escherichia coli extraintestinal infections. Clin. Microbiol. Infect. 15: 372-379.http://dx.doi.org/10.1111/j.1469-0691.2009.02708.x 